\title{
Models \& Searches of CPT Violation: a personal, very partial, list
}

\author{
Nick E. Mavromatos ${ }^{1, \star}$ \\ ${ }^{1}$ King's College London, Department of Physics, Theoretical Particle Physics and Cosmology Group, \\ Strand London WC2R 2LS, UK.
}

\begin{abstract}
In this talk, first I motivate theoretically, and then I review the phenomenology of, some models entailing CPT Violation (CPTV). The latter is argued to be responsible for the observed matter-antimatter asymmetry in the Cosmos, and may owe its origin to either Lorentz-violating background geometries, whose effects are strong in early epochs of the Universe but very weak today, being temperature dependent in general, or to an ill-defined CPT generator in some quantum gravity models entailing decoherence of quantum matter as a result of quantum degrees of freedom in the gravity sector that are inaccessible to the low-energy observers. In particular, for the latter category of CPTV, I argue that entangled states of neutral mesons (Kaons or B-systems), of central relevance to KLOE-2 experiment, can provide smoking-gun sensitive tests or even falsify some of these models. If CPT is ill-defined one may also encounter violations of the spinstatistics theorem, with possible consequences for the Pauli Exclusion Principle, which I only briefly touch upon.
\end{abstract}

\section{Introduction and Motivation}

Invariance of a relativistic (i.e. Lorentz Invariant), local and unitary field theory Lagrangian under the combined transformations of Charge Conjugation (C), Parity (spatial reflexions, (P)) and reversal in Time (T), at any order, is guaranteed by the corresponding celebrated theorem [1]. This has important implications for the equality of masses and the absolute values of the various quantum numbers that characterise particles and antiparticles, and the equal amounts of matter and antimatter when they were created in the beginning of the Universe's evolution. On the other hand, today, there is an overwhelming dominance of matter over antimatter in the Cosmos, which calls for an explanation. Assuming the validity of the CPT theorem, A. Sakharov [2] has suggested that the observed matterantimatter asymmetry in the Universe today is the result of out of thermal equilibrium processes in the early universe that violate $\mathrm{C}, \mathrm{CP}$ and Baryon number (B) symmetries. The out of equilibrium assumption is a crucial one so that any asymmetry generated by the violation of $\mathrm{C}, \mathrm{B}$ and $\mathrm{CP}$ symmetries in the expanding Universe is not washed out but remains to the current epoch, thereby explaining the observed dominance of matter over antimatter today.

Although all of Sakharov conditions are met qualitatively by the Standard Model (SM) of Particle Physics, unfortunately they are not valid quantitatively, meaning that the amount of CP violation within the SM is some ten orders of magnitude smaller than the required one to produce the observable

^e-mail: Nikolaos.Mavromatos@kcl.ac.uk 
matter-antimatter asymmetry [3]. According to observations, the abundance of baryons over that of antibaryons is of order [4]

$$
Y_{\Delta B}=\frac{n_{B}-n_{\bar{B}}}{n_{\gamma}}=(6.1 \pm 0.3) \times 10^{-10}
$$

for temperatures $T \gtrsim 1 \mathrm{GeV}$, where $n_{B}$ is the number density of baryons, $n_{\bar{B}}$ is the number density of antibaryons and $n_{\gamma}$ is the density of photons (proportional to the entropy density $s$ of the Universe). This number was determined with accurate measurements of the Cosmic Microwave Background (CMB) radiation [5]. Out of equilibrium processes that generate a baryon asymmetry in the Universe are called collectively Baryogenesis [6]. Similarly, the generation of an asymmetry between leptons. and antileptons is known as Leptogenesis [7], and is expected to be of the same order of magnitude as $Y_{\Delta B}$. Since the amount of CP violation in the SM is not sufficient to produce (1), one should look for models beyond the standard model that contain new sources of $\mathrm{CP}$ violation, that can be tuned so as to produce sufficient Baryogenesis and Leptogenesis. There is a plethora of such models in the current literature, ranging from supersymmetric theories, to theories with extra dimensions of space, including strings. There is no experimental evidence as yet for the realisation of such extended models in Nature.

Interesting models $[8,9]$ of Baryon asymmetry in the Universe involve a two stage process, during which one generates first a lepton asymmetry in the early Universe, by appropriate CP-violating non-equilibrium processes in the early Universe, which is then communicated to the baryon sector, at temperatures lower than those of the electroweak phase transition $(T \lesssim 100 \mathrm{GeV})$, by means of Baryon-minus-Lepton-(B-L)-number-conserving sphaleron processes within the SM sector of the theory. In this important scenario of Leptogenesis as the path to Baryogenesis, pioneered by Fukugita and Yanagida [8, 9], the lepton abundance is produced by the decay of heavy right-handed Majorana neutrinos (and so represents physics beyond the Standard Model (BSM)). The difference in the branching ratios of the channels of production of leptons and antileptons is equal to the imaginary part of the interference term of tree-level and one-loop diagrams for the decay processes. For the interference to generate a non-zero CP violating phase, at least two generations of right-handed neutrinos are needed [7, 8]. In fact, at least two right-handed neutrinos are also required in the see-saw mechanism [10] for the generation of light neutrino masses, as necessitated by the observed phenomena of neutrino flavour oscillations among the light neutrinos [11], which require at least two of the active neutrinos to have a mass. The model of Fukugita and Yanagida, therefore, connects an explanation of leptogenesis with the see-saw mechanism of light neutrino masses observed in Nature. The model thus represents an economical extension of SM, since, at least for the purpose of generating baryon asymmetry it does not require other particles apart from the massive right-handed neutrinos.

In the framework of ref. [8], the right handed neutrinos are very massive, and thus have decayed today, leaving no other trace apart from the see-saw type masses of the active neutrinos. Shaposhnikov and collaborators [12] have also made a proposal for a Minimal extension of the SM, termed $v M S M$, involving three generations of right-handed neutrinos, whose masses though are much lower than the corresponding ones in the model of [8]. In fact, in the $v \mathrm{MSM}$ the two heavier neutrinos are almost degenerate, with masses of order $O(1) \mathrm{GeV}$, while the lightest of the right-handed neutrinos may have masses of order of a few $\mathrm{keV}$, and has a life time longer than the age of the Universe. In this sense, the model can provide a natural candidate for dark matter (DM) ${ }^{1}$. The relatively light masses of the righthanded neutrinos in the $v \mathrm{MSM}$ makes the Baryogenesis process in this model rather complicated [14],

\footnotetext{
${ }^{1}$ In this respect, we mention that an $\mathrm{O}(50) \mathrm{keV}$ right-handed neutrino DM can play an important role in explaining galactic structures and thus resolving some of the tensions between $\Lambda \mathrm{CDM}$ model and observations at small (galactic) scales, especially if appropriate self-interactions among the keV-right-handed neutrinos are introduced [13]. The coincidence in the range of the allowed right-handed neutrino DM mass obtained in this approach, based on purely astrophysical reasons at galactic scales, with the one of the $v \mathrm{MSM}$, induced by particle physics and DM cosmology reasons, is intriguing.
} 
and certainly dissociated from the Leptogenesis path of [8]. From our point of view in this work we shall focus our attention to the model of [8], but taking into account the coupling of the fermions in the model to the gravitational background; this will have interesting consequences.

However, we shall not simply consider the Robertson-Walker Universe. Indeed, an alternative approach to baryon asymmetry, which avoids altogether the Sakharov condition on out of equilibrium processes, is the one in which from the beginning, the Universe has a matter-antimatter asymmetry, as a result of violations of the CPT symmetry. Keeping locality and unitarity intact, violation of Lorentz symmetry seems the most plausible possibility for evading the CPT theorem, and in fact this lead to a sort of anti-CPT theorem by Greenberg [15], claiming that the assumption of Lorentz symmetry plays somehow a more fundamental rôle than the other assumptions of the CPT theorem, with the conclusion that CPT violation implies necessarily a violation of Lorentz invariance. However, in his proof, Greenberg did assume the existence of a well-defined scattering transfer matrix, and in this sense his anti-CPT theorem is equivalent to the original one on CPT symmetry. Explicit counter examples to these claims, where violations of CPT occur in a Lorentz invariant way, e.g. in some non-local theories, have been provided [16]. Moreover there are Lorentz-invariant models with intrinsic decoherence [17], and it is well known that the presence of decoherence may lead [18] to an ill-defined nature of the CPT operator in the effective theory, obtained after tracing over the appropriate environmental degrees of freedom. Nevertheless, in our discussions in this talk, we shall restrict ourselves to spontaneous violation of Lorentz symmetry implying CPT Violation either through background geometries or through decoherence induced by a quantum gravity Lorentz-violating environment. In the latter case, the environmentally-induced intrinsic CPT violation may lead to interesting and unique effects in entangled states of mesons ( $\omega$-effect [19]), which we shall review at the end of talk.

The structure of the talk is the following: In section 2 we shall motivate CPT Violation (CPTV) theoretically, by considering early universe models, inspired from string theory, in which (a cosmictemperature dependent) CPTV may generate the observed matter-antimatter asymmetry in the cosmos in relatively simple models. These are much simpler than current extensions of the Standard Models, where such asymmetry requires new sources of $\mathrm{CP}$ violation. In section 3 we shall discuss the present-day phneomenology of CPTV by concentrating on models inspired by the cosmologies discussed in the previous section. We discuss stringent bounds on both, geometrically-induced CPTV, due to Lorentz Violating backgrounds, within the local effective field theory framework of the Standard Model Extension (SME) [20] (subsection 3.1), and decoherence-induced CPTV, in which the generator of the CPT transformations is ill-defined, due to information loss carried by inaccessible (to a low-energy observer) degrees of freedom of quantum gravity. This latter type of CPTV lies beyond the effective local field theory framework, and leads to characteristic evidence in entangled quantum states of neutral mesons ( $\omega$-effect, subsection 3.2). Finally, instead of conclusions, in section 4, we discuss how the latter type of CPTV, may lead to (small) spin-statistics violations.

\section{CPT Violation in Stringy Universes}

We commence our discussion on Baryon asymmetry generation by considering spontaneous violations of Lorentz symmetry, which in turn induce CPT violation ${ }^{2}$. Such violations can be provided

\footnotetext{
${ }^{2}$ For early pioneering works on matter-antimatter asymmetry generated by CPT Violating backgrounds within the SME framework we refer the reader to [21]. In that work, it was argued that, under certain circumstances, certain CPT violating terms within an SME effective Lagrangian [20] can produce large baryon asymmetry, at Grand Unified temperatures, which is eventually diluted to the current value by sphaleron processes within the SM sector. An alternative source of CPT Violating interactions that could lead to matter-antimatter asymmetry is the coupling of the baryon (or B-L) number (anomalous) current to scalar curvature $\mathcal{R}$ of space-time through a $\mathrm{CP}$ violating interaction Lagrangian $\mathcal{L}$, that could occur, e.g. within some Supergravity theories [22, 23]: $\mathcal{L}=\frac{1}{M_{\star}^{2}} \int d^{4} x \sqrt{-g}\left(\partial_{\mu} \mathcal{R}\right) J^{\mu}$, where $M_{\star}$ is a cut-off in the effective field theory and $J^{\mu}$ could be
} 
by the background geometry, for instance within the context of string-inspired models [25, 26], by the field strength of the spin-one antisymmetric tensor Kalb-Ramond field, $H_{\mu \nu \rho}=\partial_{[\mu} B_{v \rho]}$, which is totally antisymmetric in its three indices, and under certain circumstances is constant in a given frame (to be identified with the co-moving frame of the observer in an expanding Universe framework). $H_{\mu \nu \rho}$ plays the rôle of a constant totally antisymmetric torsion background field, which couples to fermions via an axial $\gamma^{\mu} \gamma^{5}$ coupling. In four space-time dimensions, $H_{\mu \nu \rho} \propto \epsilon_{\mu \nu \rho \sigma} \partial^{\sigma} b$, where $b(x)$ is a Kalb-Ramond pseudoscalar (axion-like) field. In cosmological models $b(x)=b(t)$ is only a function of the cosmic time $t$.

The constant torsion background coupling with the axial fermions implies that we should study the following model of the right-handed heavy neutrinos [26]:

$$
\mathcal{L}=i \bar{N} \not N-\frac{m}{2}\left(\overline{N^{c}} N+\bar{N} N^{c}\right)-\bar{N} \not B \gamma^{5} N-Y_{k} \bar{L}_{k} \tilde{\phi} N+\text { h.c. },
$$

where $N$ is the heavy right-handed Majorana field, satisfying the Majorana condition $N^{c}=N$, with the superscript $c$ denoting Dirac's charge conjugation, and $L_{k}$ is the lepton SU(2) left-handed doublet field of the SM, with $k$ a generation index. The adjoint of the Higgs field is defined by the relation $\tilde{\phi}_{i}=\varepsilon_{i j} \phi_{j}$. Due to our constant H-torsion situation, the axial vector background is $B^{\mu}=\dot{b} \delta^{0 \mu}$, where the overdot denotes derivative with respect to the cosmic time $t$. This is understood in what follows. For our purposes, we shall restrict ourselves [26] to only one generation of right-handed neutrinos ${ }^{3}$. Notice that the model has a portal of communication of the right-handed neutrino sector with the SM sector via the Yukawa interactions with couplings $Y_{k}$. We do not specify the mechanism for generating a mass $m$ for the heavy right-handed neutrino. The mass $m$ is determined self consistently by the requirement of the model generating sufficient leprogenesis [26].

The total lepton number asymmetry generated in this model by the tree-level decays $N \rightarrow$ $\ell^{+} \phi\left(\ell^{-} \bar{\phi}\right)$ at an appropriately calculated freeze-out temperature $T_{D}$, in the presence of a constant axial background $B_{0} \neq 0$, is estimated to be [26]:

$$
\Delta L^{T O T}=(2 r-1) n_{N}=\frac{2 \Omega B^{0}}{\Omega^{2}+\left(B^{0}\right)^{2}} n_{N} \simeq \frac{2 B^{0}}{m} n_{N}, \quad \Omega \equiv \sqrt{\left(B^{0}\right)^{2}+m^{2}} .
$$

where $n_{N}$ is the density of right-handed neutrinos $N$. The high-temperature expansion of $n_{N}$ yields for small $B_{0} \ll T_{D} \simeq m$ [26]

$$
n_{N}\left(T_{D}\right)=e^{-\beta \bar{m}}\left(\frac{\bar{m}}{2 \pi \beta}\right)^{\frac{3}{2}}+\left.O\left(B_{0}^{2}\right)\right|_{T \simeq T_{D}} \simeq 0.023 \bar{m}^{3} .
$$

The lepton asymmetry $\frac{\Delta L^{T O T}}{n_{\gamma}}$, where $n_{\gamma}$ is the photon density, is expected to be of the same order of magnitude of the baryon asymmetry (1), given that in scenarios of baryogenesis via leptogenesis we are adopting here, the asymmetry in the lepton sector is communicated to the baryon sector via Baryon (B) and Lepton (L) number violating, B-L conserving, sphaleron processes in the SM sector [3]. On making the approximation $T_{D} \simeq m$, retaining only first order terms in $\frac{B_{0}}{\bar{m}}$, and recalling that the photon number density is [28] $n_{\gamma} \simeq \frac{2 \zeta(3)}{\pi^{2}} T^{3} \simeq 0.24 T^{3}$, we obtain:

$$
\frac{B_{0}}{\bar{m}} \simeq 5 \cdot 10^{-8}, \quad \text { at } \quad T=T_{D} \simeq \bar{m} \sim 100 \mathrm{TeV} .
$$

the current associated with $B-L$ ( $L$ being the lepton number). Recently some potential problems with such models have been pointed out in [24], regarding instabilities of theories with such interactions.

${ }^{3}$ This will be sufficient for leptogenesis in our model, but of course in such a case one should consider alternative ways [27] to seesaw mechanism [10] in order to give masses to the active light neutrinos of the SM sector (that are parts of the doublet $L_{k}$ ). In case one requires a seesaw type mass generation for neutrinos, then at least two right-handed neutrino generations are needed. In such a case, there are extra sources of CP violation, which contribute to the lepton asymmetry [8]. 
If other mechanisms contributed to the lepton asymmetry in the universe, or the Yukawa couplings assume smaller values, the minimum value of $B_{0}$ would be smaller than the one given here.

In [26] the (constant) background $B^{0}$ is assumed to undergo a phase transition at temperatures near $T_{D}=100 \mathrm{TeV}$, such that

$$
B_{0}=c_{0} T^{3}, \quad c_{0}>0, \quad \text { for } \quad T \lesssim T_{D} .
$$

The parameter $c_{0}$ is a phenomenological, and can be constrained by requiring that $B^{0}$ today must be at most equal to the experimental upper bounds [29] of the $b_{0}$ (temporal) axial Lorentz and CPT violating coefficient of the Standard Model Extension [20]. Taking into account that the temperature of the Universe today (from the CMB measurements) is $T_{\mathrm{CMB}}=2.725 \mathrm{~K}=0.2348 \mathrm{meV}$, we obtain from (6) and (5) [26] $c_{0}=1 \mathrm{MeV}(100 \mathrm{TeV})^{-3}=10^{-42} \mathrm{meV}^{-2}$. implying a current value of $B_{0}$ of order

$$
B_{0 \text { today }}=O\left(10^{-44}\right) \mathrm{meV} \text {, }
$$

way too small for any experimental detection [29]. Moreover, in this case, the value of $B^{0}$ at BigBang-Nucleosynthesis $(\mathrm{BBN})$ temperature of a few $\mathrm{MeV}[28], B^{0}\left(T=T_{\mathrm{BBN}}\right) \simeq 10^{-18} \mathrm{MeV}$, is also very small, so there are negligible effects of the background on the formation of the material elements. However, in general, the critical temperature at which the phase transition for the demise of the background $B_{0}$ occurs could be significantly lower than the decoupling temperature for the right handed neutrinos, $T_{D}$; applying the experimental upper bounds on the magnitude of the background $\mathrm{H}$-torsion field $B^{0}$ today, then, we can constrain the parameter $c_{0}$ in the cooling law (6).

A different scenario for CPT-induced baryon asymmetry was discussed in [30], which is based on string Universe models with bulk D-brane defects [31]. In these models our world is a (compactified) brane Universe, with three large spatial dimensions, propagating in a higher-dimensional space bulk punctured by (inhomogeneous in general) populations of D-particle "effectively" point-like defects ${ }^{4}$. The interesting feature of this second class of models is that there is an intrinsic CPT violation in such cases, due to unobserved (by a low-energy observer) degrees of freedom associated with the recoil of the D-brane defects on the brane Universe during their interaction with string matter. In these models, CPT violation is primarily associated with an $\omega$-type effect [19], and there are different dispersion relations between particles and antiparticles as they propagate in the "medium" of D-brane defects [30], which can lead to matter-antimatter asymmetry already in thermal equilibrium.

Indeed, if $\vec{u}_{\|}$denotes the recoil velocity vector (parallel to the brane world's longitudinal directions), one may parameterise the momentum transfer during the scattering of a particle excitation off a D-particle defect by the fraction parameter of the incident momentum $r$, which is in turn assumed stochastic, that is

$$
u_{i}=\frac{g_{s}}{M_{s}} \Delta p_{i} \rightarrow g_{s} r_{i} \frac{p_{i}}{M_{s}} \quad\left(\text { no sum over i) }, \quad \ll r_{i} \gg=0, \quad \ll r_{i} r_{j} \gg=\Delta^{2} \delta_{i j} .\right.
$$

where $\ll \cdots \gg$ denotes a statistical average over D-particle populations (as well as average over quantum fluctuations of D-particles) [30]. $\Delta$ is a phenomenological parameter to be bound by experiment.

The D-foam is predominantly transparent to electrically charged matter, on account of electric charge conservation. Hence, from the SM elementary constituents of matter on the brane, mainly neutrinos and photons are interactlng dominantly with the D-particles (but in general, neutral composite particles, such as neutral mesons, are also assumed to undergo non-trivial interactions with the

\footnotetext{
${ }^{4}$ In phenomenologically relevant type IIB string theories, the D-"particles" are themselves compactified branes wrapped around circles of small radii [32], so that, from the point of view of a low-energy (with respect to the string mass scale $M_{s}$ ) observer in the brane, they look "effectively" point-like.
} 
D-foam medium, although their quark substructure may suppress them, when compared to those of neutrinos and photons. Lacking, at present, a complete theory of D-foam-matter interactions, the latter can only be studied phenomenologically). As shown in [30], the dispersion relation of the (active or right-handed) neutrino particles, of mass $m$, viewed as appropriate excitations of the open matter string, are modified due to both the metric distortion of space-time, as a result of the recoil of the D-particle, and the capture/splitting/re-emission process of the matter string by the D-particle ( $c f$. $(8))$ :

$$
\begin{aligned}
& \ll E_{v} \gg=\sqrt{p^{2}+m_{v}^{2}}\left(1+\frac{g_{s}^{2}}{2 M_{s}^{2}} \Delta^{2} p^{2}\right)-\frac{g_{s}}{2 M_{s}} \Delta^{2} p^{2} \\
& \ll \bar{E}_{\bar{v}} \gg=\sqrt{p^{2}+m_{v}^{2}}\left(1+\frac{g_{s}^{2}}{2 M_{s}^{2}} \Delta^{2} p^{2}\right)+\frac{g_{s}}{2 M_{s}} \Delta^{2} p^{2},
\end{aligned}
$$

where $\bar{E}>0$ is the (positive) energy of the antiparticle excitation. The first terms on the right-handside of these equations are due to the propagation of neutrinos in recoil-distorted space-time geometry, while the second terms are due to the capture process of a particle by the D-particle defect.

There can thus be local CPTV in the sense that the effective dispersion relation between neutrinos and antineutrinos are different in D-foam backgrounds. This difference induces a difference between neutrino and antineutrino phase-space distribution functions, which in turn generates a matterantimatter lepton asymmetry in the relevant densities

$$
\ll n-\bar{n} \gg=g_{\text {d.o.f. } \int} \int \frac{d^{3} p}{(2 \pi)^{3}} \ll[f(E)-f(\bar{E})] \gg,
$$

where $g_{\text {d.o.f. }}$ denotes the number of degrees of freedom (d.o.f.) of relativistic neutrinos and $f(E)$ is the Fermi-Dirac distribution. The lepton asymmetry can be calculated from the integrated difference (9), for $\Delta^{2}<1$, but, in contrast to conventional point-like field theory models, where the upper limit of momentum integration can be extended to $\infty$, in D-foam models, due to (8), this is extended up to the value for which the D-particle recoil velocity approaches the (upper-limiting) value of the speed of light in vacuo, $\mathrm{c}=1$ in our units, i.e. $p_{\max } \equiv|\vec{p}|_{\max }=\frac{M_{s}}{g_{s} \sqrt{\Delta^{2}}}$, where $r$ is the stochastic variable satisfying (8). The resulting integrals in (10) then become:

$$
\Delta n_{v}=\frac{g_{\text {d.o.f. }}}{2 \pi^{2}} T^{3} \int_{0}^{\frac{M_{s}}{T g_{s} \sqrt{\Delta^{2}}}} d \tilde{u}\left(\frac{1}{1+e^{\tilde{u}-\tilde{u}^{2} \frac{g_{s} \Delta^{2} T}{2 M_{s}}}}-\frac{1}{1+e^{\tilde{u}+\tilde{u}^{2} \frac{g_{s} \Delta^{2} T}{2 M_{s}}}}\right) \simeq \frac{g_{\text {d.o.f. }}}{\pi^{2}} T^{4} \frac{\Delta^{2} g_{s}}{M_{s}}
$$

The lepton asymmetry resulting from (11) freezes out at temperature $T_{d}$ and is given by:

$$
\Delta L\left(T<T_{d}\right)=\frac{\Delta n_{v}}{s}=\frac{2 \Delta^{2} g_{s} T_{d}}{M_{s}} .
$$

From (12), we observe that for a freeze-out temperature or order $T_{d} \sim 10^{15} \mathrm{GeV}$ in, say, Grand Unified Models, communicating the lepton asymmetry to baryon asymmetry via B-L preserving GUT interactions, the phenomenological value $\Delta L \sim 10^{-10}$ is attained for

$$
\frac{M_{s}}{g_{s}} \sim 10^{25} \Delta^{2} \mathrm{GeV} \text {. }
$$

For $\Delta^{2} \sim 10^{-6}$ a Planck size D-particle mass $M_{s} / g_{s} \sim 10^{19} \mathrm{GeV}$ is required so that the D-foam provides the physically observed Lepton and, thus, Baryon Asymmetry. For the unnaturally small $\Delta^{2}<10^{-21}$ one arrives at $M_{s} / g_{s} \sim 10 \mathrm{TeV}$. For $\Delta^{2} \sim O(1)$ transplanckian D-particle masses are 
required. We should stress that the above conclusions were based on specific assumptions on the freeze-out temperature.

An important remark is in order at this point. In case of right-handed Majorana neutrinos, which we discussed in the beginning of this section in connection with the model of [26], we note that they are their own antiparticles, and hence the effects of D-foam in inducing asymmetries in thermal equilibrium (10) between particles and antiparticles due to different dispersion relations do not concern them, but refer to light neutrinos that have a Dirac component in seesaw scenarios. Such asymmetries could co-exist with the mechanism of leptogenesis of [26], reviewed above, which however we would consider as the dominant one. In such models, the D-particle bulk foam could provide a way [26] for inducing the phase transition at a given era of the Universe, e.g. shortly after the decoupling of the right-handed neutrino species, that leads to the temperature-driven demise of the axial CPTV background $B_{0}(6)$, so that, in the current epoch, the value of $B^{0}$ is consistent with the stringent phenomenological bounds [29] and also with the BBN constraints.

An induced $\omega$-effect. In addition to producing matter-antimatter asymmetries, the model of [30] entails an interesting $\omega$-effect, due to decoherence associated with recoil degrees of freedom inaccessible by a low-energy observer. Indeed, consider the case of an (unnormalised) initial entangled quantum state $|i\rangle$ is given by

$$
|\psi\rangle=|k, \uparrow\rangle^{(1)}|-k, \downarrow\rangle^{(2)}-|k, \downarrow\rangle^{(1)}|-k, \uparrow\rangle^{(2)}+\xi|k, \uparrow\rangle^{(1)}|-k, \uparrow\rangle^{(2)}+\xi^{\prime}|k, \downarrow\rangle^{(1)}|-k, \downarrow\rangle^{(2)}
$$

where $\left|M_{L}(\vec{k})\right\rangle=|k, \uparrow\rangle$ in an actual situation may represent a neutral meson (Kaon $K^{0}$ or $B^{0}$ meson) and we have taken the momentum $\vec{k}$ to have only a non-zero component $k$ in the $x$-direction for brevity and concreteness; superscripts label the two separated detectors of the collinear meson pair, $\xi$ and $\xi^{\prime}$ are complex constants and we have left the state $|\psi\rangle$ unnormalised.

The effect of space-time foam on the initial entangled state of two neutral mesons, say, has been estimated in [30] by applying non-degenerate perturbation theory to the states $|k, \uparrow\rangle^{(i)},|k, \downarrow\rangle^{(i)}, i=1,2$, where the perturbation is associated with appropriate gravitational dressing of the states, due to the distortion of space-time as a result of recoil of the space-time defect, allowing for flip of the quantum numbers $\uparrow, \downarrow$ (viewed as "flavour"), during the interaction of the states with the D-particle. Assuming that the gravitationally perturbed states are still the momentum eigenstates, the dominant features of a possible $\omega$-effect can be seen from a term $\widehat{H}_{I}$ in the single-particle interaction hamiltonian [30]

$$
\widehat{H_{I}}=-\left(r_{1} \sigma_{1}+r_{2} \sigma_{2}\right) \widehat{k}, \quad \text { with } \sigma^{a}, a=1,2 \text {, the corresponding Pauli matrices , }
$$

which is the leading order contribution in the small stochastic parameters $r_{i} \ll 1$ that satisfy (cf. 8),

$$
\left\langle r_{i}\right\rangle=0, \quad\left\langle r_{i} r_{j}\right\rangle=\Delta_{i} \delta_{i j}, \quad i, j=1,2 .
$$

where we allowed for anisotropic momentum transfers in general, depending on the (left or right) direction $i=1,2$ of the neutral mesons after the decay of the initial particle in meson factories. In (16) we consider explicitly quantum averages, but statistical averages over D-particle populations should also be understood on top of the former.

In first order in perturbation theory the "gravitational dressing" of $|k, \downarrow\rangle^{(i)}$ leads to a state:

$$
\left|k^{(i)}, \downarrow\right\rangle_{Q G}^{(i)}=\left|k^{(i)}, \downarrow\right\rangle^{(i)}+\left|k^{(i)}, \uparrow\right\rangle^{(i)} \alpha^{(i)}, \quad \alpha^{(i)}=\frac{{ }^{(i)}\left\langle\uparrow, k^{(i)}\left|\widehat{H_{I}}\right| k^{(i)}, \downarrow\right\rangle^{(i)}}{E_{2}-E_{1}}
$$


and correspondingly for $\left|k^{(i)}, \uparrow\right\rangle^{(i)}$ the dressed state is obtained from (17) by exchanging $|\downarrow\rangle \leftrightarrow|\uparrow\rangle$ and $\alpha^{(i)} \rightarrow \beta^{(i i)}$ where

$$
\beta^{(i)}=\frac{{ }^{(i)}\left\langle\downarrow, k^{(i)}\left|\widehat{H_{I}}\right| k^{(i)}, \uparrow\right\rangle^{(i)}}{E_{1}-E_{2}}
$$

Here the quantities $E_{i}=\left(m_{i}^{2}+k^{2}\right)^{1 / 2}$ denote the energy eigenvalues, and $i=1$ is associated with the up state and $i=2$ with the down state. With this in mind the totally antisymmetric "gravitationallydressed" state can be expressed in terms of the unperturbed single-particle states as:

$$
\begin{aligned}
& |k, \uparrow\rangle_{Q G}^{(1)}|-k, \downarrow\rangle_{Q G}^{(2)}-|k, \downarrow\rangle_{Q G}^{(1)}|-k, \uparrow\rangle_{Q G}^{(2)}=|k, \uparrow\rangle^{(1)}|-k, \downarrow\rangle^{(2)}-|k, \downarrow\rangle^{(1)}|-k, \uparrow\rangle^{(2)} \\
& +|k, \downarrow\rangle^{(1)}|-k, \downarrow\rangle^{(2)}\left(\beta^{(1)}-\beta^{(2)}\right)+|k, \uparrow\rangle^{(1)}|-k, \uparrow\rangle^{(2)}\left(\alpha^{(2)}-\alpha^{(1)}\right) \\
& +\beta^{(1)} \alpha^{(2)}|k, \downarrow\rangle^{(1)}|-k, \uparrow\rangle^{(2)}-\alpha^{(1)} \beta^{(2)}|k, \uparrow\rangle^{(1)}|-k, \downarrow\rangle^{(2)}
\end{aligned}
$$

It should be noted that for $r_{i} \propto \delta_{i 1}$ the generated $\omega$-like effect corresponds to the case $\xi=\xi^{\prime}$ in (14) since $\alpha^{(i)}=-\beta^{(i)}$, while the $\omega$-effect of [19], specific to neutral mesons, corresponds to $r_{i} \propto \delta_{i 2}$ (and the generation of $\xi=-\xi^{\prime}$ ) since $\alpha^{(i)}=\beta^{(i)}$. In the density matrix these cases can be distinguished by the off-diagonal terms. These two cases are physically very different. In the case of $\Phi$-factories [33], the former corresponds to non-definite strangeness in the initial state of the neutral Kaons (seen explicitly when written in terms of $K_{0}-\bar{K}_{0}$ ), and hence strangeness nonconservation in the initial decay of the $\Phi$-meson, while the latter conserves this quantum number. We remind the reader that in a stochastic quantum-gravity situation, strangeness, or, in that matter, the appropriate quantum number in the case of other neutral mesons, is not necessarily conserved, and this is reflected in the above-described general parametrisation of the interaction Hamiltonian (15) in "flavour" space.

We next remark that on averaging the density matrix over the random variables $r_{i}$, we observe that only terms of order $|\omega|^{2}$ will survive, with the order of $|\omega|^{2}$ being $|\omega|^{2}=O\left(\frac{1}{\left(E_{1}-E_{2}\right)}\left(\left\langle\downarrow, k\left|H_{I}\right| k, \uparrow\right\rangle\right)^{2}\right)=$ $O\left(\frac{\Delta_{2} k^{2}}{\left(E_{1}-E_{2}\right)^{2}}\right) \sim \frac{\Delta_{2} k^{2}}{\left(m_{1}-m_{2}\right)^{2}}$ for the physically interesting case in which the momenta are of order of the rest energies (i.e. masses of the constituent states). The variance $\Delta_{2}$ (and also $\Delta_{1}$ ) is of the order of the square of the momentum transfer during the scattering of the single particle state off a space-time-foam defect ( $c f$. (9)), i.e. $\Delta_{2}=g_{s}^{2} \frac{\Delta^{2} k^{2}}{M_{s}^{2}}$, where $M_{s} / g_{s}$ the D-particle defect rest mass (with $M_{s}$ the string mass scale, and $g_{s}<1$ the (perturbative) string coupling). As already mentioned, the parameter $\Delta^{2}$ is at present a phenomenological parameter, which is proportional to the probability of interaction of the string matter with the D-particles, and therefore the total cross section of such processes. It cannot be further determined due to the lack (at present) of a complete theory of (string/brane) quantum gravity. Thus, we arrive at the following estimate of the order of $\omega$ in this model of foam [30]:

$$
|\omega|^{2} \sim g_{s}^{2} \frac{\Delta^{2} k^{4}}{M_{s}^{2}\left(m_{1}-m_{2}\right)^{2}} .
$$

In the case of neutral mesons, which (ignoring other quantum numbers, such as strangeness or beuaty) are treated as identical bosons, if the CPT operator was well defined as a quantum mechanical operator, one should have $\xi=\xi^{\prime}=0$ [34]. On the other hand, in case of quantum gravity environments, entailing loss of information for a low-energy observer, as is the case of the D-foam quantum fluctuations degrees of freedom, discussed above, the quantum generator of the CPT transformations may not be well-defined for the low-energy observer [18], which, in the case of neutral mesons, implies an initial entangled state (14) (cf. also (19)) with a non-trivial but small contamination $\xi=\xi^{\prime}=\omega \neq 0$, with $\omega=|\omega| e^{i \Omega}$ a complex parameter, so that the antiparticle state is perturbatively defined, but 
the Einstein-Podoskly-Rosen (EPR) correlations associated with the decay products of the initial entangled state (14) get modified. This is the $\omega$-effect [19], whose phenomenology will be discussed in the next section. The reader should notice that the explicit $\omega$-effect discussed in this section is Lorentz-violating, depending on the magnitude of spatial momenta of the entangled states, as a result of its origin, due to spatial recoil-velocity degrees of freedom of the D-particles, violating Lorentz symmetry locally in space and time [31].

\section{Current Phenomenology of CPT Violation}

In this section we shall discuss some aspects of the current era phenomenology of the two types of CPT Violation (CPTV) motivated by early Universe matter-antimatter asymmetry generation in the previous section. The first type of CPTV (subsection 3.1, below) is induced by Lorentz violating backgrounds, which can be studied within the framework of effective field theories, and in particular the Standard Model Extension (SME) [20] parametrization. The other CPTV type (subsection 3.2, below) goes beyond local effective field theories, and is linked to situations of environmental decoherence in which the CPT operator is not well-defined, leading to $\omega$-type modifications [19] of the Einstein-Podolsky-Rosen (EPR) correlators of entangled boson (specifically, meson) states in appropriate meson factories ( $\Phi$-factories [33] or $B$-factories [35]).

\subsection{CPT Violation within Standard Model Extension Framework}

Let us commence our discussion with the phenomenology of CPTV within the SME framework [20]. There is a phethora of precision tests which are tabularted and regularly updated in ref. [29], where we refer the interested reader for details. For our purposes here we shall only concentrate on bounds of the axial background Lorentz and CPT Violating coefficient $b_{\mu}$ in the notation of [20,29], and in particular its temporal component, which coincides with our axial background vector $B_{0}$ in (2). The first few terms in the fermion sector of the SME read [20, 29]:

$$
\begin{aligned}
\mathcal{L}_{S M E} & \ni \frac{1}{2} i \bar{\psi} \Gamma^{v} \partial_{v} \psi-\bar{\psi} \mathcal{M} \psi, \\
\mathcal{M} & \equiv m+a_{\mu} \gamma^{\mu}+b_{\mu} \gamma^{5} \gamma^{\mu}+\frac{1}{2} H^{\mu v} \sigma_{\mu \nu}, \quad \sigma_{\mu \nu}=\frac{1}{4}\left[\gamma_{\mu}, \gamma_{\nu}\right], \\
\Gamma^{v} & \equiv \gamma^{v}+c^{\mu v} \gamma_{\mu}+d^{\mu v} \gamma_{5} \gamma_{\mu}+e^{v}+i f^{v} \gamma_{5}+\frac{1}{2} g^{\lambda \mu \nu} \sigma_{\lambda \mu}, .
\end{aligned}
$$

For our purposes we note that the terms proportional to $a_{\mu}$ and $b_{\mu}$ violate both Lorentz and CPT symmetries, unlike the $c_{\mu \nu}, d_{\mu \nu}$ and $H_{\mu \nu}$ terms that violate only Lorentz symmetries. Analogous observations can be made for the various terms inside the $\Gamma^{\mu}$ structure of (21). The main assumption behind the form of such operators is that an unknown physics at high energy scales could lead to a spontaneous breaking of Lorentz invariance by giving an expectation value to certain tensorial fields, which are not in the Standard Model (SM) spectrum. The interaction of these fields with operators composed from the SM fields, which are fully Lorentz-symmetric before the spontaneous breaking, will manifest itself as effective Lorentz-Violating (LV) terms, which below the scale of the LV condensation would have the schematic form: $O_{\mu \nu \ldots}^{\mathrm{SM}} C^{\mu \nu \ldots} \rightarrow O^{\mathrm{SM}}\left\langle C^{\mu \nu \ldots}\right\rangle$, where $C^{\mu \nu \ldots}$ is an external field that undergoes condensation and $O^{\mathrm{SM}}$ is a SM field operator that transforms properly under the Lorentz group. The classification of ref. [36] requires that the independent dimension-5 operators must be gauge invariant, Lorentz invariant after contraction with the background tensors $\left\langle C^{\mu_{1} \mu_{2} \ldots}\right\rangle$, not reducible to total derivatives or to lower-dimension operators by the use of equations of motion, and they should couple to an irreducible background tensor. Several experiments, of diverse origin, can 
be used in order to impose stringent constraints on the relevant SME coefficients [29], that range from searches for forbidden atomic transitions in precision experiments and studies of low-energy antiprotonic atoms and antimatter factories, to high-energy cosmic rays, nuclear spin precession and atomic and nuclear Electric Dipole Moments (EDM) measurements, as well as data on neutrino oscillations. In the following we shall discuss some phenomenological consequences of some of the above coefficients, especially in the context of neutral mesons, forbidden transitions in (anti)hydrogen molecules or atomic dipole moment measurements. Then we shall scale the appropriate bounds on $b_{\mu}$ back in time, in the context of our CPTV stringy Universe of section 2, in order to see whether (some of) our leptogenesis scenarios discussed above can be falsified.

We commence with SME tests in antiprotonic atoms [37], in particular antihydrogen $(\overline{\mathrm{H}})$ [38]. Motivated by the theoretical microscopic models of section 2, I shall restrict myself to constraining the $b_{\mu}$ coefficients of the SME (21) using spectroscopy, in particular looking for forbidden transitions, e.g. $1 s \rightarrow 2 s$. Within $\mathrm{H}$ spectrocopic measurements, the presence of a $b_{\mu}$ coefficient in the SME (21) leads to the relevant transition of the electron in the $\mathrm{H}$ atom. The sensitivity of the tests depend crucially whether the atoms are free or trapped in an external magnetic field. In the case of free $\mathrm{H}$ (and $\overline{\mathrm{H}}$ ), the frequency shift of the 1s-2s transition is a higher-loop quantum effect in the SME/QuantumElectrodynamics (QED) lagrangian, and thus the effect is suppressed by the square of the fine structure constant, $\alpha^{2}: \delta_{1 s-2 s} \nu^{\mathrm{H}} \simeq-\alpha^{2} b_{3}^{e} / 8 \pi$, i.e. the pertinent sensitivity of such experiments would be about five orders of magnitude smaller compared to tests involving the corresponding transitions in trapped $\mathrm{H}$ and $\overline{\mathrm{H}}$. However, in the latter tests, the corresponding frequency shifts are proportional to the difference $b_{3}^{e}-b_{3}^{p}$ of the third spatial component of $b_{\mu}$ between electrons (e) and protons(p) (in a frame where the direction of the external magnetic field is along the $z$ axis). In view of the universal character of $B^{\mu}$ vectors due to background space-time geometries discussed in section 2, for this model the above difference would vanish. To cover ourselves against such cases, it is therefore imperative to either measure the sum of the coefficients $b_{\mu}^{e, p}$, or isolate them experimentally. The former can be achieved by examining hyperfine structure transitions in atomic (anti)matter. Indeed, within 1s transitions of $\mathrm{H}$ or $\overline{\mathrm{H}}$, one can determine the relevant energy shifts induced by $b_{\mu}$ [37]:

$$
\Delta_{a \rightarrow b}^{H} \simeq\left(b_{\mu}^{e}+b_{\mu}^{p}\right) / \pi+\ldots
$$

where the ... denote contributions from the rest of the SME coefficients (21), which are not written explicitly here. Hyperfine transitions within the $1 \mathrm{~S}$ level of $\mathrm{H}$ can be measured with accuracies exceeding $1 \mathrm{mHz}$ in masers. So transitions of this type in trapped $\mathrm{H}$ and $\overline{\mathrm{H}}$ are interesting candidates for performing tests of Lorentz or CPT symmetry, although to achieve resolutions of $1 \mathrm{mHz}$ in trapped antihydrogen does not seem feasible in the foreseeable future.

Another possibility would be to measure [37] radio-frequency transitions between states within the triplet of hyperfine levels in $\mathrm{H}$ and $\overline{\mathrm{H}}$, in particular the so called $|d\rangle_{1} \rightarrow|c\rangle_{1}$ transition at external magnetic fields of order $\mathrm{B} \simeq 0.65$ Tesla. The corresponding frequency shifts depend solely on $b_{3}^{p}$ :

$$
\Delta_{c \rightarrow d}^{\mathrm{H}} \simeq-b_{3}^{p} / \pi, \quad \Delta_{c \rightarrow d}^{\overline{\mathrm{H}}} \simeq+b_{3}^{p} / \pi
$$

where we took into account that under the action of CPT operation, which exchanges $\mathrm{H}$ and $\overline{\mathrm{H}}$, the coefficient of the $b_{3}^{p}$ changes sign. Thus, comparison of the above spectroscopic measurement between trapped $\mathrm{H}$ and $\overline{\mathrm{H}}$ would yield immediately a bound (or a measurement!) on $b_{3}^{p}$. If a frequency resolution of $1 \mathrm{mHz}$ could be attained (which at present is far from being plausible), then, one could obtained $\left|b_{3}^{p}\right| \leq 10^{-27} \mathrm{GeV}$. Still such bounds are about four orders of magnitude smaller that the ones coming from masers. We also note that, although, clock-comparison experiments are able to resolve spectral lines to about $1 \mu \mathrm{Hz}$, nevertheless, isolating $b_{i}^{p}$ is very complicated due to the complex structure of the nuclei involved. 
The above experiments are sensitive only to spatial components of Lorentz-violating couplings. Sensitivity to timelike couplings, $b^{0}$, would require appropriate boosts. On the other hand, in the context of the model (2) of section 2, such experiments can bound the combinations $\gamma \vec{v}_{3} B^{0}$, where $\vec{v}$ is the current-era relative velocity of us (as local observers) with respect to the CMB (or FriedmannRobertson-Walker) frame. Currently, we can quote the following bounds on $b_{\mu}$ coefficients for electrons [29]:

$$
b^{0} \leq 0.02 \mathrm{eV}, \quad|\vec{b}| \leq 10^{-21} \mathrm{eV} .
$$

It should be mentioned that the stringent limits on $|\vec{b}|$ have been obtained in measurements using torsion pendulum containing macroscopic numbers of polarised electrons [39]. New interactions, such as the above-mentioned Lorentz- and CPT-violating ones are then searched for (and bounded) by looking at the corresponding effects on the electron spin. Such tests may also be performed in man-made antihydorgen [38] or other anti-atoms, with the aim of providing direct comparison of CPT properties and thus tests of CPT invariance.

Next we describe the situation governing the constraints on the relevant dimension- 5 terms of the SME lagrangian coming from EDM. These are generically found to be of order [36] $\leq 10^{-25} e \mathrm{~cm}$. The overall expression for the total EDM, due to the CP Violating conventional QED terms and the CPT Violating terms due to the presence of an appropriate Lorentz-violating background vector $n^{\mu}$, is obtained from the effective Lagrangian

$$
\mathcal{L}_{\mathrm{EDM}}=-i \frac{1}{2} d_{\mathrm{CP}} \bar{\psi} \sigma^{\mu v} F_{\mu \nu}(A) \psi+d_{\mathrm{CPT}} \bar{\psi} \gamma^{\mu} \gamma^{5} F_{\mu v}(A) n^{v} \psi
$$

where $F_{\mu v}$ is the Maxwell field strength. The currently null result on the neutron dipole moment imposes the constraint $d_{\mathrm{CP}}+d_{\mathrm{CPT}}=0$. The lagrangian (25) should be completed with the $a^{\mu}$ and $b^{\mu}$ SME terms (21), as well as the appropriate dimension-5 operators from the QED sector of the SME [36]:

$$
\mathcal{L}_{5}=\sum_{\text {fermion species }}\left[c^{\mu} \bar{\psi} \gamma^{\lambda} F_{\lambda \mu} \psi+d^{\mu} \bar{\psi} \gamma^{\lambda} \gamma^{5} F_{\lambda \mu} \psi+g^{\mu} \bar{\psi} \gamma^{\lambda} \tilde{F}_{\lambda \mu} \psi+f^{\mu} \bar{\psi} \gamma^{\lambda} \gamma^{5} \tilde{F}_{\lambda \mu} \psi\right],
$$

where $\tilde{F}_{\mu \nu}$ is the dual of the Maxwell tensor. The various terms in (26) have different transformation properties under the action of the discrete symmetries $\mathrm{C}, \mathrm{P}$ and $\mathrm{T}$, which, together with the corresponding terms of (21), are indicated in the table of fig. 1, on the assumption that the vector backgrounds are time-like and invariant under C,P and T reflections [36]. Experimentally [40], one can disentangle CP-odd from CPT-odd operators, because of different suppression scales. Specifically, the former require helicity flip and are thus represented by dimenion-six operators in the SME effective lagrangian, with suppression by the $\mathrm{CP}$ breaking scale of order $1 / \Lambda_{\mathrm{CP}}^{2}$. Such operators imply spin precession in a magnetic field relative to the direction of $\mathbf{B} \times \mathbf{v}$. On the other hand, the CPT-odd operators are of dimension 5 , as they do not require helicity flip, e.g. in the quark sector such operators are of the form $\bar{q}_{R(L)} \gamma^{v} \gamma^{5} F_{\nu \mu} q_{R(L)}$, and $\bar{q}_{L} \gamma^{v} \gamma^{5} F_{v \mu}^{a} \tau^{a} q_{L}$, where $\tau^{a}, a=1,2,3$ are the SU(2) generators of the weak interaction standard model group, and $F_{\mu \nu}$ and $F_{\mu \nu}^{a}$ are the U(1) and SU(2) gauge field strengths respectively. These operators are suppressed linearly by the CPT-breaking scale, $1 / \Lambda_{\mathrm{CPT}}$.

EDMs have been bounded with high precision in several occasions [40]: (i) neutrons, with the bound $d_{n}<3 \times 10^{-26} \mathrm{e} \mathrm{cm}$, (ii) diamagnetic atoms (such as $\mathrm{Hg}, \mathrm{Xe}, \ldots$ ), for which the corresponding EDMs are induced by the EDMs of the valence nucleons (for the case of mercury EDM, one has the (approximate) relation: $d_{\mathrm{Hg}} \simeq-5 \times 10^{-4}\left(d_{n}+0.1 d_{p}\right) \sim-5 \times 10^{-4} d_{n}$, which implies that a signal consistent with CPT violation would occur, if non-zero $d_{n}, d_{\mathrm{Hg}}$ were to be found), and (iii) paramagnetic atoms (such as Tl, Cs, ...): their EDM are extremely suppressed as a result of the absence of a CPT-odd electron EDM. 


\begin{tabular}{cc||ccc}
\hline Coefficient & Operator & $C$ & $P$ & $T$ \\
\hline$a^{0}$ & $\bar{\psi} \gamma_{0} \psi$ & - & + & + \\
$b^{0}$ & $\bar{\psi} \gamma_{0} \gamma_{5} \psi$ & + & - & + \\
$c^{0}$ & $F_{\lambda 0} \bar{\psi} \gamma^{\lambda} \psi$ & + & + & - \\
$d^{0}$ & $F_{\lambda 0} \bar{\psi} \gamma^{\lambda} \gamma^{5} \psi$ & - & - & - \\
$f^{0}$ & $\widetilde{F}_{\lambda 0} \bar{\psi} \gamma^{\lambda} \gamma^{5} \psi$ & - & + & + \\
$g^{0}$ & $\widetilde{F}_{\lambda 0} \bar{\psi} \gamma^{\lambda} \psi$ & + & - & + \\
\hline
\end{tabular}

Figure 1. Tranformation properties of the various terms in (26) under the action of the discrete symmetries $\mathrm{C}, \mathrm{P}$ and T. $\mathrm{A}+(-)$ indicates an even (odd) function. From ref. [36].

In general, theoretical estimates of dimension-three operators induced by multiloop CP violating corrections in the standard model, imply the following bounds of the SME coefficients in (21) [40]

$$
a^{\mu}, b^{\mu} \sim d^{\mu}\left(10^{-20}-10^{-18}\right) \mathrm{GeV}^{2},
$$

providing sensitivity to $d^{\mu} \leq 10^{-12} \mathrm{GeV}^{-1}$ and thus $\Lambda_{\mathrm{CPT}} \sim\left(10^{11}-10^{12}\right) \mathrm{GeV}$, if one takes into account the current bounds on $b_{\mu}$ [29] (cf. (24)).

Higher LV background tensors, e.g. terms in SME effective lagrangian of the form $\mathcal{D}_{\mu \nu \rho} \bar{e} \gamma^{\rho} \gamma^{5} e F_{\mu \nu}$, can also be bounded experimentally with high accuracy, by looking [40] for corrections to the spin precession frequency of the form $\left(\mathcal{D}^{i[0 k]}+\mathcal{D}^{k[0 i]}\right) E_{i} B_{k}$, which changes sign under the reversal of the electric field $E_{i}$. The relative signal changes during the day as a result of the change of the Laboratory orientation relative to the tensor background. We also mention the interesting suggestion of ref. [41] on further tests of CPT symmetry due to the CPT-odd axial vector background $b^{\mu}$, which has been of interest to us in section 2. According to this work, within the framework of LV extended electrodynamics, the Dirac equation for a bound electron in an external electromagnetic field has been considered, assuming the interaction with the background field $b_{\mu}$. A Foldy-Wouthysen quasi-relativistic (1/c)-series expansion (truncated to order $1 / c^{2}$ ) has been applied to obtain an effective Hamiltonian for the hydrogen atom and through this the relativistic Dirac eigenstates in a spherically-symmetric potential to second order in $b^{0}$. The $b^{0}$-induced CPT-odd corrections to the electromagnetic dipole moment operators of a bound electron have been calculated. Such corrections contribute to the anapole moment of the atomic orbital and may cause a specific asymmetry of the angular distribution of the radiation of a hydrogen atom, in particular the $2 p_{1 / 2,1 / 2} \rightarrow 1 s_{1 / 2,-1 / 2}$ ( $c f$. fig. 2). The non-observation currently of such asymmetries leads to bounds of the magnitude of $\left|b^{0}\right|$ : $\left|b^{0}\right| \leq 2 \times 10^{-8} m_{e} c^{2} \simeq 10^{-11} \mathrm{GeV}$, which are consistent with the general bounds (24) for the SME coefficient $b_{\mu}$ for electrons [29].

Finally we mention that, further tests of CPT invariance can be made by direct measurements of particle antiparticle mass and charge differences, which we are not going to discuss here. However, in the spirit of our cosmological model discussed in section 2, we do mention that, if the observed 


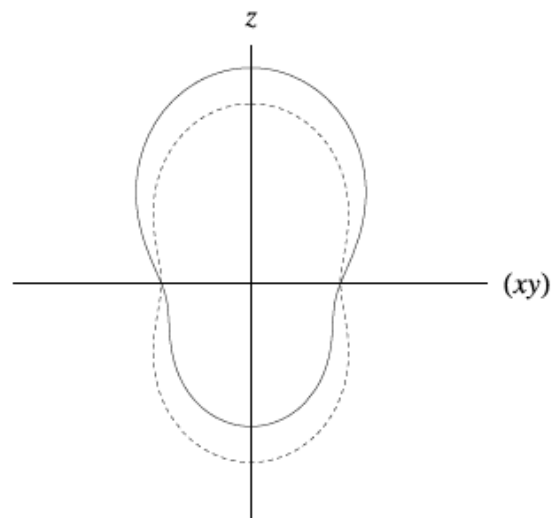

Figure 2. Anglular distribution for spontaneous radiation for the atomic transition $2 p_{1 / 2,1 / 2} \rightarrow 1 s_{1 / 2,-1 / 2}$ in the presence of a CPT-odd SME axial background vector $b^{\mu}$. The dashed line indicates the standard electrodynamics $b^{\mu}=0$ case. From ref. [41].

matter/antimatter asymmetry were due to a mass difference between particle and antiparticles, then, one may make the reasonable assumption that baryogenesis could be due to mass differences between quarks and antiquarks [42]. The latter nay depend linearly with temperature, $m_{q}(T) \sim g T$, as a consequence of known high-temperature properties of Quantum Chromodynamics (QCD). Furthermore, it is reasonable (although not strictly necessary) to assume that the quantk-antiquark differences today are bound by the current bound on proton-antiproton mass difference, which is of order $7 \times 10^{-10}$ $\mathrm{GeV}$, as provided in 2011 by the ASACUSA Collaboration [38]. Scaling back in temperature such differences, up to the respective decoupling temperature of the quarks, lead to baryon asymmetries that are much smaller than the observed one [42].

In this sense the model of [25] can still survive, given that, even if a $B^{0}<0.02 \mathrm{eV}$ is observed today, according to the current SME limits, the Universe may have undergone such a (or series of) phase transition at $T \sim 10^{9} \mathrm{GeV}$ towards a smaller (or zero) H-torsion background. This is an (crude) example of how one can use current SME bounds to fit early universe cosmologies. In a similar spirit, the model of [26] on leptogenesis through CPT and CP violating decays of heavy right-handed neutrinos, discussed in section 2, based on the Lagrangian (2), with the assumption that the critical temperature of the phase transition that triggers the demise of the axial background $B_{0}$ is of the same order as the decoupling temperature of right-handed neutrinos, $T_{D} \sim 100 \mathrm{TeV}$ (5), lies comfortably within the limits (24), given the scaling (6) of the background $B^{0}=b^{0}$ and its current value (7). Reversing the logic, we can assume the upper bound of $B^{0}$ today (24), scale back in time with the scaling (6) up to BBN temperatures, $T_{\mathrm{BBN}} \sim O(1) \mathrm{MeV}$, and then constrain the coefficient $c_{0}$ by the requirement that the $\mathrm{BBN}$ conditions are not disturbed. Then. one can continue scaling back in the cosmic time, to check at which temperature range sufficient leptogenesis is produced, if at all. Such procedures require of course detailed models of baryogenesis via leptogenesis, which we reserve for future studies. 


\subsection{Intrinsic CPT Violation in Quantum Decoherence Models - the $\omega$-effect}

We now come to examine the current phenomenology of the $\omega$-effect [19], which is associated with a second type of CPTV, already discussed in the context of the D-foam model in section 2, in which the quantum CPT operator is not well-defined. We stress again that this latter type of CPTV goes beyond local effective field theories, and its most sensitive bounds can be placed in experimental facilities involving entangled states of neutral mesons, such as neutral $\operatorname{Kaon}(\Phi)$ factories $[33,43]$ or $B-\bar{B}$ meson factories [35].

In case of loss of information for a low energy observer, carried by d.o.f. inaccessible to him/her due to quantum gravity environments (e.g. the gravitational reocoil d.o.f. in the D-foam example of section 2), the quantum operator that generates CPT symmetry may not be well defined [18]. A proof of this statement is obtained by recuctio ad absurdum, that is by first assuming the existence of a well-defined unitary and invertible CPT operator acting on density matrices (antiunitary if acting on state vectors): $\Theta$, such that (a bar above an operator denotes a quantity pertaining to anti-matter states, obtained via the action of the CPT transformation):

$$
\rho_{\text {out }}(t \rightarrow \infty)=\$ \bar{\rho}_{\text {in }}(t \rightarrow-\infty)
$$

where the subscript "in" and "out" denotes asymptotic states, and the density matrix is defined as $\rho=$ $\operatorname{tr}|\psi\rangle\langle\psi|$, where the trace operation "tr" is over quantum states inaccessible to a low energy observer. The "superscattering matrix" $\$$ is a linear operator acting on density matrices without an inverse, due to the existance of information loss in the problem. We may then write (from now one we ignore the time $t$ arguments of the asymptotic $\rho$, for brevity):

$$
\rho_{\text {out }}=\$ \rho_{\text {in }} \Rightarrow \Theta-\rho_{\text {in }}=\$ \Theta^{-1} \bar{\rho}_{\text {out }} \Rightarrow \bar{\rho}_{\text {in }}=\Theta^{-1} \$ \Theta^{-1} \bar{\Theta}_{\text {out }} .
$$

However, since $\bar{\rho}_{\text {out }}=\$ \bar{\rho}_{\text {in }}$, the last relation on the right-hand-side of (29) implies

$$
\bar{\rho}_{\text {in }}=\Theta^{-1} \$ \Theta^{-1} \$ \bar{\rho}_{\text {in }} .
$$

But this is impossible, as it would imply that the superscattering operator $\$$ has an inverse $\Theta^{-1} \$ \Theta^{-1}$, that contradicts the initial assumption of information loss ${ }^{5}$. This is the so called strong form of (intrinsic) CPT violation, which would imply a microscopic time arrow.

Nevertheless in nature there could be [18] a weak form of CPT invariance, according to which the microscopic time arrow does not show in any scattering experiments. Indeed, in such a case the experimentalist would be able to prepare initial pure quantum mechanical state vectors, and there should a well defined transition probability $\mathcal{P}$ from the initial pure state $|\psi\rangle$ to the final state $|\phi\rangle$, such that

$$
\mathcal{P}(\psi \rightarrow \phi)=\mathcal{P}\left(\theta^{-1} \phi \rightarrow \theta \psi\right)
$$

where the (antiunitary) CPT operator $\theta$ acts on "in" and "out" Hilbert spaces $\mathcal{H}$ vectors now, $\theta$ : $\mathcal{H}_{\text {in }} \rightarrow \mathcal{H}_{\text {out }}$, and is such that

$$
\Theta \rho=\theta \rho \theta^{\dagger}, \quad \theta^{\dagger}=-\theta^{-1},
$$

which in terms of the $\$$ matrix can be written as

$$
\$^{\dagger}=\Theta^{-1} \$ \Theta^{-1} \text {. }
$$

Whether there exists such a situation of weak form of CPT invariance is in general an experimental question. The $\omega$-effect [19] is one way to answer this question experimentally, and, as we have seen

\footnotetext{
${ }^{5}$ In a theory without information loss, of course, $\$$ would factorise to the Heisenberg scattering matrix $S$ as $\$=S S^{\dagger}$, and would have a well-defined inverse, $\$^{-1}=\$^{\dagger}$, in which case the CPT operator would be well defined satisfying $\Theta=\$ \Theta^{-1} \$$.
} 
in 2, it characterises the D-foam example. We next proceed to the phenomenology of this effect in entangled states of mesons, which, if observed, would constitute a "smoking-gun" evidence for such an intrinsic CPT violation.

We commence our discussion by briefly mentioning direct tests of Time reversal invariance, independent of CP and CPT violation, within the Lorentz invariant Standard Model theory, using entangled neutral mesons. Eventually we shall use those tests assuming the presence of a CPTV $\omega$-effect in order to constrain the real and imaginary parts of the corresponding parameter. These tests have been initially proposed in [44], leading to the recent observation of direct $\mathrm{T}$ violation by the Ba-Bar collaboration [35], through the exchange of initial and final states in transitions that can only be connected by a T -symmetry transformation. For example, the transition $\bar{B}^{0} \rightarrow B^{-}$for the second $\mathrm{B}$ to decay, at time $t_{2}$, once the first B (entangled with the second) has been tagged at time $t_{1}$, is identified by reconstructing events in the time-ordered final states $\left(\ell^{+} X, J / \psi K_{s}^{0}\right)$. The rate of this transition is then compared to that of the $B^{-} \rightarrow \bar{B}^{0}$ transition, that exchanges initial and final states, which is identified by the reconstruction of the final states $\left(J / \psi K_{L}^{0}, \ell^{-} X\right)$. Any observed difference between these two rates, would thus indicate direct observation of $\mathrm{T}$ violation, independent of $\mathrm{CP}$ properties [45]. This would also imply an independent test of CPT symmetry within the Standard Model. Similar tests of T violation in entangled Kaon $\Phi$ factories have also been suggested [46], by identifying the appropriate reactions that exchange initial and final states. However, if CPT is intrinsically violated, in the sense of being not well defined due to decoherence [18] induced by quantum gravity [47], the above-mentioned direct observation of $\mathrm{T}$ violation cannot constitute a test of decoherence-induced CPT breaking. This is because in such a case a distinct phenomenon, associated with the ill-defined nature of CPT operator, emerges, termed $\omega$-effect [19]. If the $\omega$ effect were present, such direct Tviolation tests using entangled states of B-mesons [48] would allow the experimenter to disentangle it from conventional CPT violating effects in the Hamiltonian, within the SME framework, and also to measure independently $\operatorname{Im} \omega$ and $\operatorname{Re} \omega$. We shall comment briefly on this later in the section.

For the moment, let us concentrate first to the neutral Kaon system, where the $\omega$-effects are dominant, as compared to other entangled neutral mesons, although conceptually our analysis applies equally [49] to entangled B-meson factories as well, such as those of [35]. In a quantum-gravity induced decohered situation, the Neutral mesons $K^{0}$ and $\bar{K}^{0}$ should no longer be treated as identical particles. As a consequence [19], the initial entangled state in $\Phi$ factories $|i\rangle$, after the $\Phi$-meson decay, assumes the form:

$$
|i\rangle=\mathcal{N}\left[\left(\left|K_{S}(\vec{k}), K_{L}(-\vec{k})\right\rangle-\left|K_{L}(\vec{k}), K_{S}(-\vec{k})\right\rangle\right)+\omega\left(\left|K_{S}(\vec{k}), K_{S}(-\vec{k})\right\rangle-\left|K_{L}(\vec{k}), K_{L}(-\vec{k})\right\rangle\right)\right],
$$

where $\omega=|\omega| e^{i \Omega}$ is a complex parameter, parametrizing the intrinsic CPTV modifications of the EPR correlations [19]. The $\omega$-parameter controls the amount of contamination of the final C(odd) state by the "wrong" (C(even)) symmetry state. The appropriate observable (c.f. fig. 3) is the "intensity" $I(\Delta t)=\int_{\Delta t \equiv\left|t_{1}-t_{2}\right|}^{\infty}|A(X, Y)|^{2}$, with $A(X, Y)$ the appropriate $\Phi$ decay amplitude [19], where one of the Kaon products decays to the final state $X$ at $t_{1}$ and the other to the final state $Y$ at time $t_{2}$ (with $t=0$ the moment of the $\Phi$ decay).

It must be noted that in Kaon factories there is a particularly good channel, the one with bi-pion states $\pi^{+} \pi^{-}$as final decay products, which enhances the sensitivity to the $\omega$-effect by three orders of magnitude. This is due to the fact that the relevant terms [19] in the intensity $I(\Delta t)$ (c.f. fig. 3) contain the combination $\omega /\left|\eta_{+-}\right|$, where $\eta_{+-}$is the relevant CP-violating amplitude for the $\pi^{+} \pi^{-}$states, which is of order $10^{-3}$. The KLOE experiment bounds of the $\omega$ parameter are [43]:

$$
\operatorname{Re}(\omega)=\left(-1.6_{-2.1 \text { stat }}^{+3.0} \pm 0.4_{\text {syst }}\right) \times 10^{-4}, \quad \operatorname{Im}(\omega)=\left(-1.7_{-3.0 \text { stat }}^{+3.3} \pm 1.2_{\text {syst }}\right) \times 10^{-4}
$$




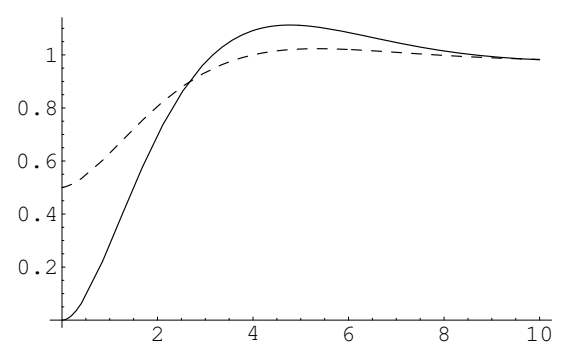

Figure 3. A characteristic case of the intensity $I(\Delta t)$ (vertical axis) as a function of $\Delta t$ (horizontal axis), with $|\omega|=0$ (solid line) vs $I(\Delta t)$ (dashed line) with $|\omega|=\left|\eta_{+-}\right|, \Omega=\phi_{+-}-0.16 \pi$, for definiteness [19].

At least an order of magnitude improvement is expected for upgraded facilities such as KLOE-2 at (the upgraded) DAФNE-2 [43].

This sensitivity is not far from certain optimistic models of space time foam leading to $\omega$-like effects [30]. Indeed, let us comparing these bounds to the D-foam case (20). First, we recall that successful leptogenesis from this class of models requires heavy D-particles masses (13). Assuming for definiteness D-particle masses of order of the Planck mass, $M_{s} / g_{s} \sim M_{P}$, we recall that the variance $\Delta^{2} \sim O\left(10^{-6}\right)(13)$ in order to have phenomenolgically acceptable leptogenesis. In the case of neutral kaons, with momenta of the order of the rest energies $(\sim 1 \mathrm{GeV})$ and mass differences $m_{1}-m_{2} \simeq 10^{-15} \mathrm{GeV}$, we observe from (20) that $|\omega| \sim 10^{-4}|\Delta|$ (whilst for $B$-mesons we have $\left.|\omega| \sim 10^{-6}|\Delta|\right)$. For $1>\Delta \geq 10^{-3}$ these values for $\omega$ are not far below the sensitivity of current facilities, such as KLOE-2 at DAФNE, and thus such leptogenesis CPTV models may be constrained experimentally in the foreseeable future.

In B-factories one can look for similar $\omega$-like effects. Although in this case there is no particularly good channel to lead to enhancement of the sensitivity, as in the $\Phi$-factories, nevertheless one gains in statistics, and hence interesting limits may also be obtained [49]. The presence of a quantum-gravity induced $\omega$-effect in B systems is associated with a theoretical limitation on flavour tagging, namely the fact that in the absence of such effects the knowledge that one of the two-mesons in a meson factory decays at a given time through a flavour-specific channel determines unambiguously the flavour of the other meson at the same time. This is not true if intrinsic CPT Violation is present. One of the relevant observables [49] is given by the CP-violating semi-leptonic decay charge asymmetry (in equal-sign dilepton channel), with the first decay $B \rightarrow X \ell^{ \pm}$being time-separated from the second decay $B \rightarrow X^{\prime} \ell^{ \pm}$by an interval $\Delta t$. In the absence of $\omega$-effects, the intensity at equal decay times vanishes, $I_{\mathrm{sl}}\left(\ell^{ \pm}, \ell^{ \pm}, \Delta t=0\right)=0$, whilst in the presence of a complex $\omega=|\omega| e^{i \Omega}, I_{\mathrm{sl}}\left(\ell^{ \pm}, \ell^{ \pm}, \Delta t=0\right) \sim$ $|\omega|^{2}$. In such a case, the asymmetry observable exhibits a peak, whose position depends on $|\omega|$, while the shape of the curve itself depends on the phase $\Omega$ [49]. The analysis of [49], using the above charge asymmetry method and comparing with currently available experimental data, leads to the following bounds:

$$
-0.0084 \leq \operatorname{Re}(\omega) \leq 0.0100, \quad \text { at } 95 \% \text { C.L. . }
$$

Such tests for intrinsic CPT violation may be performed simultaneously with the above-mentioned observations of direct T violation, as they are completely independent. Quite recently, we have embarked [48] on a detailed study of $\omega$-effects in $B_{d}$-system, using the experimental procedure suggested in [44], and implemented in [35], for tests of T violation in etnangled meson systems independent of $\mathrm{CP}$ violation. We have identified how to probe the complex $\omega$ parameter in the entangled $B_{d}$-system using Flavour(f)-CP(g) eigenstate decay channels: the connection between the Intensities for the two 
time-ordered decays (f, g) and $(\mathrm{g}, \mathrm{f})$ is lost in the presence of a non-zero $\omega$. Appropriate observables have been constructed allowing independent experimental determinations of $\operatorname{Re}(\omega)$ and $\operatorname{Im}(\omega)$, disentangled from CPT violation in the evolution Hamiltonian, $\operatorname{Re}(\theta)$ and $\operatorname{Im}(\theta)$, which parametrise CPTV within the SME local effective field theory frameworks. The general analysis of [48] has found

$$
\begin{array}{rll}
\operatorname{Im}(\omega) & = \pm(6.40 \pm 2.80) \times 10^{-2}, & \operatorname{Re}(\omega)=(1.09 \pm 1.60) \times 10^{-2} \\
\operatorname{Im}(\theta) & = \pm(6.11 \pm 3.45) \times 10^{-2}, & \operatorname{Re}(\theta)=(0.99 \pm 1.98) \times 10^{-2}
\end{array}
$$

where the $2.4 \sigma$ deviations from $\operatorname{Im}(\omega)=0$ and $\operatorname{Re}(\theta)=0$ are interpreted as upper bounds. These $2 \sigma$ tensions have been shown to be uncorrelated [48].

At this juncture, I would like to point out that an observation of the $\omega$-effect in both the $\Phi$ and B-factories could also provide an independent test of Lorentz symmetry properties of the intrinsic CPT Violation, namely whether the effect respects Lorentz symmetry. This is because, although the $\Phi$ particle in neutral Kaon factories is produced at rest, the corresponding $\Upsilon$ state in B-factories is boosted, and hence there is a frame change between the two experiments. If the quantum gravity $\omega$ effect is Lorentz violating, as is the case of the models considered in section 2 [30], then a difference in the value of $\omega$ between the two experiments should be expected.

Finally, since Lorentz Violation has been mentioned, I also point out that bounds of the LV SME coefficients $a^{\mu}$ ( $c f$. eq. (21)) can be placed by measurements in the entangled Kaon $\Phi$ factories [43]. In particular by adopting the relevant SME terms to the quark sector, relevant for Kaon physics, one can bound differences $\Delta a^{\mu}=a_{q_{1}}^{\mu}-a_{q_{2}}^{\mu}$, where $q_{i}, i=, 2$ denote appropriate quark states. The current experimental limits for the coefficients $\Delta_{a}^{\mu}$ are: from the KTeV Collaboration $\Delta_{X}, \Delta a_{Z}<9.2 \times$ $10^{-22} \mathrm{GeV}$, while from the the KLOE Collaboration in the DaФNE $\Phi$ factory [43] are less competitive but with the advantage that entangled meson factories have sensitivity to all four coefficients $\Delta a^{\mu}$, in particular: $\Delta a^{0}=(0.4 \pm 1.8) \times 10^{-17} \mathrm{GeV}$, from KLOE, with expected sensitivity at KLOE-2 in upgraded DAФNE facilties for $\Delta a^{X, Y, Z}=O\left(10^{-18}\right) \mathrm{GeV}$. Unfortunately, entangled meson factories have only sensitivity to differences $\Delta a^{\mu}$ rather than absolute coefficients $a^{\mu}$. Of course, if gravity acts universally for all quark species, such differences may be zero.

\section{Instead of Conclusions : Intrinsic CPTV and Spin-Statistics Theorem}

Before closing the talk I would like to make some remarks on another potential effect of quantumgravity-decoherence-induced CPTV, namely tiny violations of the Spin-Statistics theorem [1] and in particular Pauli exclusion principle and related searches [50]. The Spin-Statistics Theorem states that the wave function of a system of identical integer-spin particles has the same value when the positions of any two particles are swapped, while the wave function of a system of identical half-integer spin particles changes sign when two particles are swapped. Particles with wave functions symmetric under exchange are called bosons and those with wave functions antisymmetric under exchange are called fermions. The theorem was first proposed by Fierz in 1939, by Pauli in a more systematic formulation in 1940, and in a rigorous mathematical formalism, using quantum field theory path integrals, by Schwinger in 1950, where the underlying mathematical assumptions were made clear:

- (1) The theory has a Lorentz and CPT invariant Lagrangian and relativistic causality.

- (2) The vacuum is Lorentz-invariant (can be weakened).

- (3) The particle is a localized excitation. Microscopically, it is not attached to a string or domain wall.

- (4) The particle is propagating (has a non-infinite mass). 
- (5) The particle is a real excitation, meaning that states containing this particle have a positivedefinite norm and has positive energy.

An important consequence of the spin-statistics theorem is that the wavefunction of two identical fermions is zero, hence two identical fermions (i.e. with all quantum numbers the same) cannot occupy the same state, which is the celebrated Pauli exclusion Principle (PEP), that was postulated by Pauli in 1925, without knowledge of the spin-statistics theorem at the time.

We should remark at this point that when a violation of the spin-statistics theorem appears, it is one or more of the above assumptions that they are violated. For instance, spinless anticommuting fields, which could exist in condensed matter models are not relativistic invariant; ghost fields in gauge theories are spinless fermions but they have negative norm. The $2+1$ dimensional Chern-Simons theory has anyons (fractional spin excitations) but in such a case the wave function of the planar system splits between the bulk and the boundary, and hence is somehow delocalised. One remark concerns quarks: Despite being attached to a confining string, QCD quarks can have a spin-statistics relation proven at short distances (ultraviolet limit) due to asymptotic freedom.

We have mentioned above that CPT and Lorentz invariance are crucial assumptions for the validity of the spin-statistics theorem. Although spontaneous violation of Lorentz and CPT symmetry may be tolerated (see assumption (2), which may be relaxed), nevertheless if the CPT is ill defined, and there is a strong (intrinsic) form of CPT violation, as is the case of quantum-gravity induced decoherence (or D-foam situations, as in section 2), then there may be tiny violations of the spin-statistics theorem. In this respect, we recall that it was the Bose-Statistics requirement of the neutral mesons that resulted in the antisymmetric initial state (34) when $\omega=0$. In D-foam situations [31], for instance, the matter excitation is dressed by open strings stretched between the defect and the brane world, and in this sense, assumption (3) of the theorem is violated, together with assumption (1), due to the ill-defined nature of the CPT operator. In such cases, there are hidden degrees of freedom in a particle state, and thus an evasion, in case of fermions, of the PEP may be understood by the fact that the otherwise looking identical quantum states entering the PEP formulation, actually differ by hidden quantum numbers. Thus we believe that searches for intrinsic CPT violation could also be complemented by searches of PEP. The current searches of PEP [50] are mainly for charged particles (electrons), for which, as we have discussed previously [32], the effects of the D-particle foam are expected to be strongly suppressed. Nevertheless, this is only one model and experiment should be independent of any theoretical assumptions, hence searches of PEP are equally encouraged as searches of intrinsic CPT violation.

\section{Acknowledgements}

I thank the organisers of KLOE-2 2016 Workshop on $e^{+}-e^{-}$collisions physics at $1 \mathrm{GeV}$ (Frascati Lab. INFN (Italy), October 26-28 2016) for their kind invitation to give a talk and for stimulating discussions. The work of N.E.M. is partially supported by STFC (UK) via the research grant ST/L000326/1.

\section{References}

[1] R. F. Streater and A. S. Wightman, PCT, spin and statistics, and all that (Princeton, USA: Princeton Univ. Press) and references therein.

[2] A. D. Sakharov , Pisma Zh. Eksp. Teor. Fiz. 5, 32 (1967) [JETP Lett. 5, 24 (1967)] [Sov. Phys. Usp. 34, 392 (1991)] [Usp. Fiz. Nauk 161, 61 (1991)].

[3] V. A. Kuzmin, V. A. Rubakov and M. E. Shaposhnikov , Phys. Lett. B 155, 36 (1985); M. B. Gavela, P. Hernandez, J. Orloff and O. Pene , Mod. Phys. Lett. A 9, 795 (1994) 
doi:10.1142/S0217732394000629 [hep-ph/9312215]; M. B. Gavela, P. Hernandez, J. Orloff, O. Pene and C. Quimbay, Nucl. Phys. B 430, 382 (1994) doi:10.1016/0550-3213(94)00410-2 [hep-ph/9406289].

[4] For a review see: W. Buchmuller, R. D. Peccei and T. Yanagida, Ann. Rev. Nucl. Part. Sci. 55, 311 (2005) [hep-ph/0502169], and references therein.

[5] D. N. Spergel et al. [WMAP Collaboration] , Astrophys. J. Suppl. 148, 175 (2003) [astroph/0302209]. P. A. R. Ade et al. [Planck Collaboration] , Astron. Astrophys. 571, A16 (2014) [arXiv:1303.5076 [astro-ph.CO]].

[6] R. N. Mohapatra and P. B. Pal, World Sci. Lect. Notes Phys. 60, 1 (1998) [World Sci. Lect. Notes Phys. 72, 1 (2004)]. M. Trodden, Rev. Mod. Phys. 71, 1463 (1999) [hep-ph/9803479]; W. Buchmuller, arXiv:0710.5857 [hep-ph].

[7] M. C. Chen, TASI 2006 Lectures on Leptogenesis (2007), hep-ph/0703087 [HEP-PH]. A. Pilaftsis, J. Phys. Conf. Ser. 171, 012017 (2009) [arXiv:0904.1182 [hep-ph]]; J. Phys. Conf. Ser. 447, 012007 (2013).

[8] M. Fukugita and T. Yanagida, Phys. Lett. B 174, 45 (1986).

[9] M. A. Luty, Phys. Rev. D 45, 455 (1992).

[10] P. Minkowski, Phys. Lett. B 67, 421 (1977); M. Gell-Mann, P. Ramond and R. Slansky, in Supergravity, eds. D.Z. Freedman and P. van Nieuwenhuizen (North-Holland, Amsterdam, 1979); T. Yanagida, in Proc. of the Workshop on the Unified Theory and the Baryon Number in the Universe (Tsukuba, Japan, eds. O. Sawada and A. Sugamoto, 1979); R. N. Mohapatra and G. Senjanovic, Phys. Rev. Lett. 44, 912 (1980); J. Schechter and J. W. F. Valle, Phys. Rev. D 22, 2227 (1980); G. Lazarides, Q. Shafi and C. Wetterich, Nucl. Phys. B 181, 287 (1981); doi:10.1016/0550-3213(81)90354-0

[11] M. C. Gonzalez-Garcia and M. Maltoni, Phys. Rept. 460, 1 (2008) [arXiv:0704.1800 [hep-ph]].

[12] T. Asaka, S. Blanchet and M. Shaposhnikov, Phys. Lett. B 631, 151 (2005); doi:10.1016/j.physletb.2005.09.070 [hep-ph/0503065]. For cosmological/astrophysical applications of this model see: A. Boyarsky, O. Ruchayskiy and M. Shaposhnikov, Ann. Rev. Nucl. Part. Sci. 59, 191 (2009) doi:10.1146/annurev.nucl.010909.083654 [arXiv:0901.0011 [hep-ph]]; M. Shaposhnikov, Prog. Theor. Phys. 122, 185 (2009). doi:10.1143/PTP.122.185

[13] C. R. Argüelles, N. E. Mavromatos, J. A. Rueda and R. Ruffini, JCAP 1604, no. 04, 038 (2016) doi:10.1088/1475-7516/2016/04/038 [arXiv:1502.00136 [astro-ph.GA]]; N. E. Mavromatos, C. R. Argüelles, R. Ruffini and J. A. Rueda, Int. J. Mod. Phys. D 26, no. 03, 1730007 (2016); doi:10.1142/S0218271817300075, and references therein.

[14] L. Canetti, M. Drewes, T. Frossard and M. Shaposhnikov, Phys. Rev. D 87, 093006 (2013) doi:10.1103/PhysRevD.87.093006 [arXiv:1208.4607 [hep-ph]].

[15] O. W. Greenberg, Phys. Rev. Lett. 89, 231602 (2002) doi:10.1103/PhysRevLett.89.231602 [hep$\mathrm{ph} / 0201258]$.

[16] M. Chaichian, K. Fujikawa and A. Tureanu, Phys. Lett. B 712, 115 (2012) doi:10.1016/j.physletb.2012.04.048 [arXiv:1203.0267 [hep-th]]; Eur. Phys. J. C 73, no. 3, 2349 (2012) doi:10.1140/epjc/s10052-013-2349-2 [arXiv:1205.0152 [hep-th]].

[17] G. J. Milburn, New J. Phys. 8, 96 (2006) doi:10.1088/1367-2630/8/6/096 [gr-qc/0308021].

[18] R. M. Wald, Phys. Rev. D 21, 2742 (1980) doi:10.1103/PhysRevD.21.2742

[19] J. Bernabeu, N. E. Mavromatos and J. Papavassiliou, Phys. Rev. Lett. 92, 131601 (2004) doi:10.1103/PhysRevLett.92.131601 [hep-ph/0310180]; E. Alvarez, J. Bernabeu, N. E. Mavromatos, M. Nebot and J. Papavassiliou, Phys. Lett. B 607, 197 (2005) doi:10.1016/j.physletb.2004.12.032 [hep-ph/0410409]; J. Bernabeu, N. E. Mavro- 
matos, J. Papavassiliou and A. Waldron-Lauda, Nucl. Phys. B 744, 180 (2006) doi:10.1016/j.nuclphysb.2006.03.028 [hep-ph/0506025].

[20] D. Colladay and V. A. Kostelecky, Phys. Rev. D 58, 116002 (1998) [hep-ph/9809521].

[21] O. Bertolami, D. Colladay, V. A. Kostelecky and R. Potting, Phys. Lett. B 395, 178 (1997) doi:10.1016/S0370-2693(97)00062-2 [hep-ph/9612437].

[22] H. Davoudiasl, R. Kitano, G. D. Kribs, H. Murayama and P. J. Steinhardt, Phys. Rev. Lett. 93, 201301 (2004) doi:10.1103/PhysRevLett.93.201301 [hep-ph/0403019].

[23] B. Mukhopadhyay, Mod. Phys. Lett. A 20, 2145 (2005) doi:10.1142/S0217732305017640 [astro-ph/0505460]. U. Debnath, B. Mukhopadhyay and N. Dadhich, Mod. Phys. Lett. A 21, 399 (2006) doi:10.1142/S0217732306019542 [hep-ph/0510351]. G. Lambiase and S. Mohanty, Phys. Rev. D 84, 023509 (2011) doi:10.1103/PhysRevD.84.023509 [arXiv:1107.1213 [hep-ph]]. G. Lambiase, Phys. Lett. B 642, 9 (2006) doi:10.1016/j.physletb.2006.09.037 [hep-ph/0612212]. S. Capozziello and G. Lambiase, Frascati Phys. Ser. 58, 17 (2014) [arXiv:1409.3370 [gr-qc]]. J. I. McDonald and G. M. Shore, Phys. Lett. B 751, 469 (2015) doi:10.1016/j.physletb.2015.10.075 [arXiv:1508.04119 [hep-ph]]. Phys. Lett. B 766, 162 (2017) doi:10.1016/j.physletb.2016.11.057 [arXiv:1604.08213 [hep-ph]].

[24] E. V. Arbuzova and A. D. Dolgov, arXiv:1702.07477 [gr-qc]; arXiv:1612.06206 [gr-qc].

[25] J. Ellis, N. E. Mavromatos and S. Sarkar, Phys. Lett. B 725, 407 (2013) doi:10.1016/j.physletb.2013.07.016 [arXiv:1304.5433 [gr-qc]].

[26] M. de Cesare, N. E. Mavromatos and S. Sarkar, Eur. Phys. J. C 75, no. 10, 514 (2015) doi:10.1140/epjc/s10052-015-3731-z [arXiv:1412.7077 [hep-ph]].

[27] N. E. Mavromatos and A. Pilaftsis, Phys. Rev. D 86, 124038 (2012) doi:10.1103/PhysRevD.86.124038 [arXiv:1209.6387 [hep-ph]].

[28] E. W. Kolb and M. S. Turner, The Early Universe, Front. Phys. 69, 1-547.

[29] V. A. Kostelecky and N. Russell, Data Tables for Lorentz and CPT Violation, Rev. Mod. Phys. 83, 11 (2011) doi:10.1103/RevModPhys.83.11 [arXiv:0801.0287 [hep-ph]].

[30] J. Bernabeu, N. E. Mavromatos and S. Sarkar, Phys. Rev. D 74, 045014 (2006) doi:10.1103/PhysRevD.74.045014 [hep-th/0606137]; N. E. Mavromatos and S. Sarkar, Eur. Phys. J. C 73, no. 3, 2359(2013) doi:10.1140/epjc/s10052-013-2359-0 [arXiv:1211.0968 [hep$\mathrm{ph}]$.

[31] J. R. Ellis, N. E. Mavromatos and M. Westmuckett , Phys. Rev. D 70, 044036 (2004) doi:10.1103/PhysRevD.70.044036 [gr-qc/0405066]; ibid. Phys. Rev. D 71, 106006 (2005) doi:10.1103/PhysRevD.71.106006 [gr-qc/0501060]; J. R. Ellis, N. E. Mavromatos, D. V. Nanopoulos and M. Westmuckett, Int. J. Mod. Phys. A 21, 1379 (2006) doi:10.1142/S0217751X06028990 [gr-qc/0508105]; N. E. Mavromatos, V. A. Mitsou, S. Sarkar and A. Vergou, Eur. Phys. J. C 72, 1956 (2012) doi:10.1140/epjc/s10052-012-1956-7 [arXiv:1012.4094 [hep-ph]]; N. E. Mavromatos, M. Sakellariadou and M. F. Yusaf, JCAP 1303, 015 (2013) doi:10.1088/1475-7516/2013/03/015 [arXiv:1211.1726 [hep-th]]; T. Elghozi, N. E. Mavromatos, M. Sakellariadou and M. F. Yusaf, JCAP 1602, no. 02, 060 (2016) doi:10.1088/1475-7516/2016/02/060 [arXiv:1512.03331 [hep-th]].

[32] T. Li, N. E. Mavromatos, D. V. Nanopoulos and D. Xie, Phys. Lett. B 679, 407 (2009) doi:10.1016/j.physletb.2009.07.062 [arXiv:0903.1303 [hep-th]].

[33] A. Di Domenico, Search for CPT and Lorentz-Symmetry Violation in Entangled Neutral Kaons, arXiv:1608.00241 [hep-ph]; A. De Santis [KLOE-2 Collaboration and DAFNE Team], DAФNE and KLOE-2, Phys. Scripta T 166, 014015 (2015) doi:10.1088/0031-8949/2015/T166/014015 [arXiv:1503.06002 [physics.ins-det]] and references therein. 
[34] J. Bernabeu, F. J. Botella and J. Roldan, FTUV/89-35, IFIC/89-11, Proc. Blois Conference on CP Violations, pp. 389-400 (1989)

[35] J. P. Lees et al. [BaBar Collaboration], Phys. Rev. Lett. 109, 211801 (2012) [arXiv:1207.5832 [hep-ex]]; G. Eigen [BaBar Collaboration], Recent BABAR Results, J. Phys. Conf. Ser. 631, no. 1, 012033 (2015) doi:10.1088/1742-6596/631/1/012033 [arXiv:1503.02867 [hep-ex]] and references therein.

[36] P. A. Bolokhov and M. Pospelov, Phys. Rev. D 77, 025022 (2008) [hep-ph/0703291 [HEP-PH]], and references therein.

[37] N. Russell, hep-ph/0209251; M. Hori and J. Walz, Prog. Part. Nucl. Phys. 72, 206 (2013) doi:10.1016/j.ppnp.2013.02.004 [arXiv:1304.3721 [physics.atom-ph]], and references therein.

[38] M. Hori et al. [ASACUSA Collaboration], Nature 475, 484 (2011); doi:10.1038/nature10260; M. Hori et al. [ASACUSA Collaboration], Science 354, no. 6312, 610 (2016) doi:10.1126/science.aaf6702

[39] B. R. Heckel, E. G. Adelberger, C. E. Cramer, T. S. Cook, S. Schlamminger and U. Schmidt, Phys. Rev. D 78, 092006 (2008) [arXiv:0808.2673 [hep-ex]].

[40] P. A. Bolokhov, M. Pospelov and M. Romalis, Phys. Rev. D 78, 057702 (2008) [hep$\mathrm{ph} / 0609153$ ] and references therein.

[41] O. G. Kharlanov and V. C. .Zhukovsky, J. Math. Phys. 48, 092302 (2007) [arXiv:0705.3306 [hep-th]].

[42] A.D. Dolgov, Phys. Atom. Nucl., 73, 588 (2010), arXiv: 0903.4318, and references therein.

[43] F. Ambrosino et al. [KLOE Collaboration], Phys. Lett. B 642, 315 (2006); A. Di Domenico, arXiv:1608.00241 [hep-ph].

[44] M. C. Banuls and J. Bernabeu, Nucl. Phys. B 590, 19 (2000) [hep-ph/0005323]; J. Bernabeu, M. C. Banuls and F. Martinez-Vidal, PoS HEP 2001, 057 (2001) [hep-ph/0111073]; J. Bernabeu, F. Martinez-Vidal and P. Villanueva-Perez, JHEP 1208, 064 (2012) [arXiv:1203.0171 [hep-ph]]; See also: E. Alvarez and A. Szynkman, Mod. Phys. Lett. A 23, 2085 (2008) [hep-ph/0611370].

[45] J. Bernabeu, F. J. Botella and M. Nebot, JHEP 1606, 100 (2016) doi:10.1007/JHEP06(2016)100 [arXiv:1605.03925 [hep-ph]].

[46] J. Bernabeu, A. Di Domenico and P. Villanueva-Perez, Nucl. Phys. B 868, 102 (2013) [arXiv:1208.0773 [hep-ph]].

[47] J. R. Ellis, J. S. Hagelin, D. V. Nanopoulos and M. Srednicki, Nucl. Phys. B 241, 381 (1984); J. R. Ellis, J. L. Lopez, N. E. Mavromatos and D. V. Nanopoulos, Phys. Rev. D 53, 3846 (1996); P. Huet and M. E. Peskin, Nucl. Phys. B 434, 3 (1995); F. Benatti and R. Floreanini, Nucl. Phys. B 488, 335 (1997).

[48] J. Bernabeu, F. J. Botella, N. E. Mavromatos and M. Nebot, arXiv:1612.05652 [hep-ph].

[49] E. Alvarez, J. Bernabeu, N. E. Mavromatos, M. Nebot and J. Papavassiliou, Phys. Lett. B 607, 197 (2005); E. Alvarez, J. Bernabeu and M. Nebot, JHEP 0611, 087 (2006).

[50] J. Marton et al. , arXiv:1703.01615 [quant-ph]; A. Pichler et al., J. Phys. Conf. Ser. 718, no. 5, 052030 (2016) doi:10.1088/1742-6596/718/5/052030 [arXiv:1602.00898 [physics.insdet]]; C. Curceanu et al., Found. Phys. 46, 263 (2016) doi:10.1007/D10701-015-9923-4, 10.1007/s10701-015-9923-4 [arXiv:1601.06617 [quant-ph]]. H. Shi et al. [VIP Collaboration], J. Phys. Conf. Ser. 718, no. 4, 042055 (2016) doi:10.1088/1742-6596/718/4/042055 [arXiv:1601.05828 [physics.ins-det]]. C. Curceanu et al., J. Phys. Conf. Ser. 631, no. 1, 012068 (2015) doi:10.1088/1742-6596/631/1/012068. 